\title{
O OSCE na avaliação clínica odontológica: relato de experiência com estudantes de graduação
}

Gustavo de Almeida Logar*; Cláudia de Oliveira Lima Coelho**; Eliane Cristina Gava Pizi***; Graziela Ávila Prado Galhano ****; Adrieli de Paula Neves*; Ligia Teixeira de Oliveira***; José Maria Bertão***

* Mestre, Professor Assistente, Faculdade de Odontologia, UNOESTE

** Mestre em Educação, Diretora da Faculdade de Odontologia, UNOESTE

*** Doutor, Professor Assistente, Faculdade de Odontologia, UNOESTE

***** Pós-doutorado, Professor Assistente, Faculdade de Odontologia, UNOESTE

Recebido em 29/05/2017. Aprovado em 10/09/2017.

\section{RESUMO}

O Exame Clínico Objetivo e Estruturado (OSCE) é um exame organizado em estações que simulam a realidade clínica e os estudantes são avaliados em tarefas específicas como anamnese, exame físico, comunicação com paciente, procedimentos clínicos não invasivos, entre outras. É realizado o rodízio de alunos e um ou dois examinadores pontuam o desempenho dos mesmos em cada tarefa, com critérios e tempo previamente determinados. O objetivo desse estudo foi relatar a experiência de aplicação do OSCE na graduação e como estruturá-lo. O primeiro OSCE aplicado pelo curso de Odontologia da Universidade do Oeste Paulista (UNOESTE) para alunos de graduação foi no ano de 2016, com o objetivo de avaliação de competências dos alunos que ingressariam nas clínicas no semestre seguinte. A avaliação foi aplicada aos 70 alunos do final do terceiro ano e os conteúdos selecionados foram Dentística, Periodontia, Endodontia, Radiologia, Biossegurança, Anestesiologia e Diagnóstico Bucal. O OSCE pode ser considerado uma técnica de avaliação adequada para o alcance de competências clínicas na formação de cirurgiões-dentistas, por proporcionar ao aluno de graduação a vivência de atividades similares à realidade que enfrentará no atendimento clínico e em sua futura atuação profissional. Esse método de avaliação propicia aos estudantes a oportunidade de serem avaliados de forma mais objetiva e poder aprender com o feedback do seu desempenho no exame.

Descritores: Avaliação. Educação em Odontologia. Odontologia. 


\section{INTRODUÇÃO}

O mundo atual vive inúmeras modificações na área do conhecimento, da tecnologia, nas áreas sociopolítica e da saúde, que avançam com enorme rapidez. É necessário um novo perfil de profissional que atua na área da saúde: generalista, humanista, crítico e reflexivo; cuja prática seja pautada na ética, na ciência, na integralidade, na cidadania e na promoção de saúde ${ }^{1}$.

As tendências atuais de ensino em saúde apontam para a utilização de metodologias ativas com o objetivo de buscar o aprendizado do aluno em que o professor é um facilitador do conhecimento e, dessa forma, é possível a existência da articulação entre a assistência e o ensino, voltando-se para determinantes biopsicossociais do processo saúde e doença dessa comunidade e para inserção da tecnologia da informação no processo de formação .

Para avaliar a qualidade profissional e as competências clínicas do estudante, a observação dos princípios da pirâmide de Miller devem ser contemplados na escolha dos métodos de avaliação. $\mathrm{O}$ aluno precisa saber (conhecimento), saber como faz (relatar, descrever), realizar em situações simuladas (demonstrar como faz) e também o grau mais elevado da pirâmide (fazer) ${ }^{3,4}$.

Como não existe um único método de avaliação capaz de atingir todos estes elementos (conhecimentos, habilidades e atitudes), entendese que apenas a combinação de métodos é capaz de produzir os resultados esperados da avaliação ${ }^{5}$.

O Exame Clínico Objetivo e Estruturado (OSCE) é um exame organizado em estações que simulam a realidade clínica e os alunos são avaliados em tarefas específicas como anamnese, exame físico, comunicação com paciente, procedimentos clínicos não invasivos, entre outras. É realizado o rodizio de alunos e um ou dois examinadores pontuam o desempenho dos mesmos em cada tarefa, com critérios e tempo previamente estruturados ${ }^{6-8}$.

Assim, este exame é fundamental para a identificação de lacunas nas habilidades clínicas dos alunos e corrigir estas deficiências. Além disso, esta avaliação permite que os alunos reflitam sobre seu desempenho, com feedback imediato de seus erros e acertos ${ }^{8-10}$.

O OSCE foi inserido inicialmente na Medicina e, em outros países, tem sido realizado na Odontologia. No Brasil este método avaliativo é aplicado em poucos cursos de graduação em Odontologia. Nestes cursos o OSCE é utilizado como ferramenta de avaliação pré-clínica associado às avaliações teóricas e laboratoriais para verificar se o aluno apresenta o mínimo de habilidades e conhecimentos para o atendimento clínico de pacientes ${ }^{9,11-13}$.

As avaliações clínicas e pré-clínicas desempenham quatro papéis essenciais na formação do cirurgião-dentista: 1) fornecer dados para a auto avaliação dos estudantes, 2) informar aos professores sobre o sucesso de seu ensino, 3) certificar a competência dos alunos e 4) assegurar a qualidade do cuidado à saúde oferecida aos pacientes nas clínicas odontológicas das universidades e na atuação profissional depois de formado $^{2,14}$.

A fase pré-clínica é importante, pois o treinamento é realizado em manequins que simulam as condições clínicas que os alunos enfrentarão no atendimento dos pacientes. Esse treinamento serve para que a teoria faça sentido na prática, para que o estudante aprimore suas habilidades motoras e sinta-se seguro para atender pacientes reais nas clínicas do curso ${ }^{15-17}$. 
Qualquer conteúdo ministrado nas disciplinas pré-clínicas pode ser avaliado no OSCE, desde que não seja um procedimento invasivo, mesmo em pacientes que necessitem deste atendimento. No entanto, alguns destes procedimentos invasivos podem ser simulados em manequins utilizados nos laboratórios préclínicos, em mandíbulas e línguas de animais como boi e porco ${ }^{18-20}$.

Além disso, o OSCE pode ser considerado um exame que segue a maioria dos critérios de uma boa avaliação, como validade (afere o grau no qual o teste realmente avalia aquilo que propõe), confiabilidade (afere a reprodutibilidade e a consistência de um teste), viabilidade e efeito catalisador (promoção de resultados e feedback produzindo melhor suporte educacional $)^{21,22}$. Esses critérios foram analisados em estudos realizados por Eberhard ${ }^{9}$ e Graham $^{10}$ que, após aplicação de OSCE na graduação de Odontologia e análise estatística dos dados, concluíram que esse exame pode ser válido, confiável e viável para o uso em cursos de Odontologia.

Tendo em vista as diversas possibilidades avaliativas do método e a pouca utilização nos cursos de Odontologia brasileiros, o objetivo desse estudo foi relatar a experiência de aplicação do OSCE na graduação e de como estruturá-lo.

\section{RELATO DE EXPERIÊNCIA}

O primeiro OSCE aplicado pelo curso de Odontologia da Universidade do Oeste Paulista (UNOESTE) para alunos de graduação foi no ano de 2016, com o objetivo de avaliação das competências dos estudantes que ingressariam no ciclo clínico no semestre seguinte.

A avaliação foi aplicada aos 70 alunos do final do terceiro ano e os conteúdos selecionados para o OSCE foram Dentística, Periodontia, Endodontia, Radiologia, Biossegurança, Anestesiologia e Diagnóstico Bucal.

Para o planejamento da estrutura do OSCE foi criada uma comissão de avaliação, com reuniões periódicas mensais para decidir sobre as tarefas, checklists, lista de materiais, recrutamento de equipe de apoio e de avaliadores, avaliação do espeço físico para a realização do exame, logística de confinamento de alunos pré e pós avaliação, assim como feedback.

O desenvolvimento do OSCE foi dividido em fases: determinação do conteúdo, desenvolvimento das estações, programa de ação prévio ao OSCE, programa de ação no dia do OSCE e programa de ação após o término do OSCE.

\section{Determinação do conteúdo: Blueprinting}

O primeiro passo no desenvolvimento de um OSCE é determinar o que deve ser avaliado, como as habilidades clínicas, de comunicação, diagnóstico e o conhecimento exigido do aluno para atuar nas disciplinas clínicas e em sua vida profissional. O importante é que o estudante tenha entrado em contato com o conteúdo na teoria ou na prática antes de ser avaliado.

A quantidade de estações a serem desenvolvidas depende do objetivo do OSCE, do tempo e espaços disponíveis e do número de alunos a ser avaliados.

No OSCE realizado na UNOESTE foram elaboradas estações para 7 componentes curri-culares, realizado para 70 alunos com 8 horas de duração e 1h30min de feedback, totalizando 9h30min.

Para cada um dos 7 componentes curriculares foram elaboradas duas estações diferentes, pois metade da turma de alunos realizou o exame no período da manhã e a outra metade no período da tarde. 


\section{Desenvolvimento das estações}

É importante que o material que faz parte das estações seja listado com grande antecedência à aplicação do exame, para que possa ser revisado e testado antes da avaliação.

Para cada estação, após a decisão do objetivo da tarefa (figura 1), foram elaboradas as instruções ao aluno sobre a tarefa a ser realizada. $\mathrm{O}$ estudante teria um minuto para leitura das instruções e cinco minutos para a execução das atividades propostas. Desta forma, as orientações deveriam ser claras e sucintas para permitir a leitura e reflexão dentro do período estipulado. Em seguida foi elaborada a lista de materiais necessários para a montagem de cada estação.

\section{Programa de ação prévio ao OSCE}

Para a realização do OSCE, deve-se iniciar pela seleção do local adequado e neste caso optouse pelos boxes das clínicas odontológicas da faculdade.

Uma segunda etapa foi o recrutamento dos examinadores com instrução de como preencher o checklist (figura 2), padronização da avaliação e como proceder ofeedback ao final do exame. Este checklist apresentava os itens que o avaliador precisava observar na execução das tarefas pelo aluno e para cada quesito uma pontuação foi estabelecida: tarefa completa $(1,0)$, tarefa parcialmente completa $(0,5)$ e tarefa não realizada $(0,0)$ com nota final de 0 a 10 .

Além disso, para a elaboração de algumas estações foi necessário o recrutamento de pacientes simulados e manequins. Nas estações de Semiologia e Radiologia foi selecionado o paciente simulado para diagnóstico de queixa dolorosa e para posicionamento na tomada radiográfica, respectivamente. Para aumentar a confiabilidade do teste, garantindo que todos os alunos experimentem a mesma atuação foi necessário o treinamento do ator para a explicação detalhada do papel, leitura e discussão do roteiro escrito, inclusão de modificações pertinentes, memorização, avaliação do entendimento da situação e domínio do roteiro, simulações e correções com avaliador para ajustes finais.

Por fim, o uso de manequim das práticas laboratoriais odontológicas foi utilizado na simulação do procedimento de posicionamento da matriz na área de Dentística.

\begin{tabular}{|c|c|c|}
\hline Estações & Manhã & Tarde \\
\hline Anestesiologia & $\begin{array}{l}\text { Seleção de sal anestésico para extração } \\
\text { dentária em gestante }\end{array}$ & $\begin{array}{l}\text { Seleção de sal anestésico para extração } \\
\text { dentária em paciente hipertenso }\end{array}$ \\
\hline Biossegurança & Lavagem correta das mãos & Colocação de barreiras de proteção \\
\hline Dentística & $\begin{array}{c}\text { Seleção de matriz para restauração em } \\
\text { amálgama }\end{array}$ & $\begin{array}{l}\text { Seleção de matriz para restauração em } \\
\text { resina }\end{array}$ \\
\hline Endodontia & Odontometria do dente 21 & Odontometria do dente 24 \\
\hline Semiologia & $\begin{array}{l}\text { Identificação de cadeia de linfonodos } \\
\text { cervicais }\end{array}$ & Hipótese diagnóstica para lesão de boca \\
\hline Radiologia & $\begin{array}{l}\text { Simulação de técnica radiográfica pela } \\
\text { bissetriz }\end{array}$ & Simulação da técnica de Clark \\
\hline Periodontia & $\begin{array}{l}\text { Seleção de instrumentais para raspagem } \\
\text { dos dentes } 23 \text { e } 37\end{array}$ & $\begin{array}{l}\text { Seleção de instrumentais para raspagem } \\
\text { dos dentes } 12 \text { e } 44\end{array}$ \\
\hline
\end{tabular}

Figura 1. Estações utilizadas no OSCE 
O OSCE na avaliação clínica odontológica: relato de experiência com estudantes de graduação

\begin{tabular}{|c|c|c|c|c|}
\hline Disciplina: Dentística & \multicolumn{3}{|c|}{ Disciplina: Dentística clínica } & Período: Sexto Termo \\
\hline Estação: Clínica & \multicolumn{3}{|c|}{ Professor: } & Data: \\
\hline \multicolumn{5}{|c|}{ CHECKLIST } \\
\hline \multicolumn{5}{|l|}{ Aluno: } \\
\hline \multicolumn{5}{|l|}{ Avaliador: } \\
\hline TAREFA & Fez & $\begin{array}{c}\text { Fez } \\
\text { parcial }\end{array}$ & Não fez & Comentários \\
\hline 1. Realizou adequada paramentação para procedimento & 1 & 0,5 & 0 & \\
\hline 2. Posicionou-se / ergonomia & 1 & 0,5 & 0 & \\
\hline $\begin{array}{l}\text { 3. Indicou corretamente a matriz necessária para o } \\
\text { procedimento }\end{array}$ & 1 & 0,5 & 0 & \\
\hline $\begin{array}{l}\text { 4. Segurou a matriz metálica seccional com pinça clínica e a } \\
\text { inseriu cuidadosamente }\end{array}$ & 1 & 0,5 & 0 & \\
\hline $\begin{array}{l}\text { 5. Adaptação da matriz. Verificou altura e projeção na } \\
\text { cervical. Deixou a tira matriz em posição adequada }\end{array}$ & 1 & 0,5 & 0 & \\
\hline $\begin{array}{l}\text { 6. Após posicionar a matriz, inseriu a cunha entre a matriz e } \\
\text { face proximal do dente adjacente }\end{array}$ & 1 & 0,5 & 0 & \\
\hline $\begin{array}{l}\text { 7. Cunhas inseridas pelas maiores ameias. Verificar se não } \\
\text { inseriu a cunha invertida. }\end{array}$ & 1 & 0,5 & 0 & \\
\hline 8. Utilizou o anel de afastamento & 1 & 0,5 & 0 & \\
\hline $\begin{array}{l}\text { 9. Boa adaptação da matriz e da cunha. Verificar: } \\
\text { altura/projeção na cervical e concavidade; adaptação da } \\
\text { matriz na região cervical e se toca no dente adjacente. }\end{array}$ & 1 & 0,5 & 0 & \\
\hline $\begin{array}{l}\text { 10. Fez a brunidura complementar da tira matriz contra o } \\
\text { dente adjacente. Verificar a adaptação da matriz na região } \\
\text { cervical e se toca no dente adjacente. }\end{array}$ & 1 & 0,5 & 0 & \\
\hline TOTAL DE PONTOS (máximo 10 pontos) & & & & \\
\hline
\end{tabular}

Figura 2. Modelo de checklist

Revista da ABENO • 18(1):15-24, 2018 - DOI: https://doi.org/10.30979/rev.abeno.v18i1.444 


\section{Programa de ação no dia do OSCE}

No final de semana anterior à avaliação foi realizada a montagem das estações, verificando quais boxes serviriam de estação, fixação dos números das estações e das tarefas impressas na parede do box, e colocação dos materiais e manequins para uso pelo aluno nas tarefas (figura 3 ).

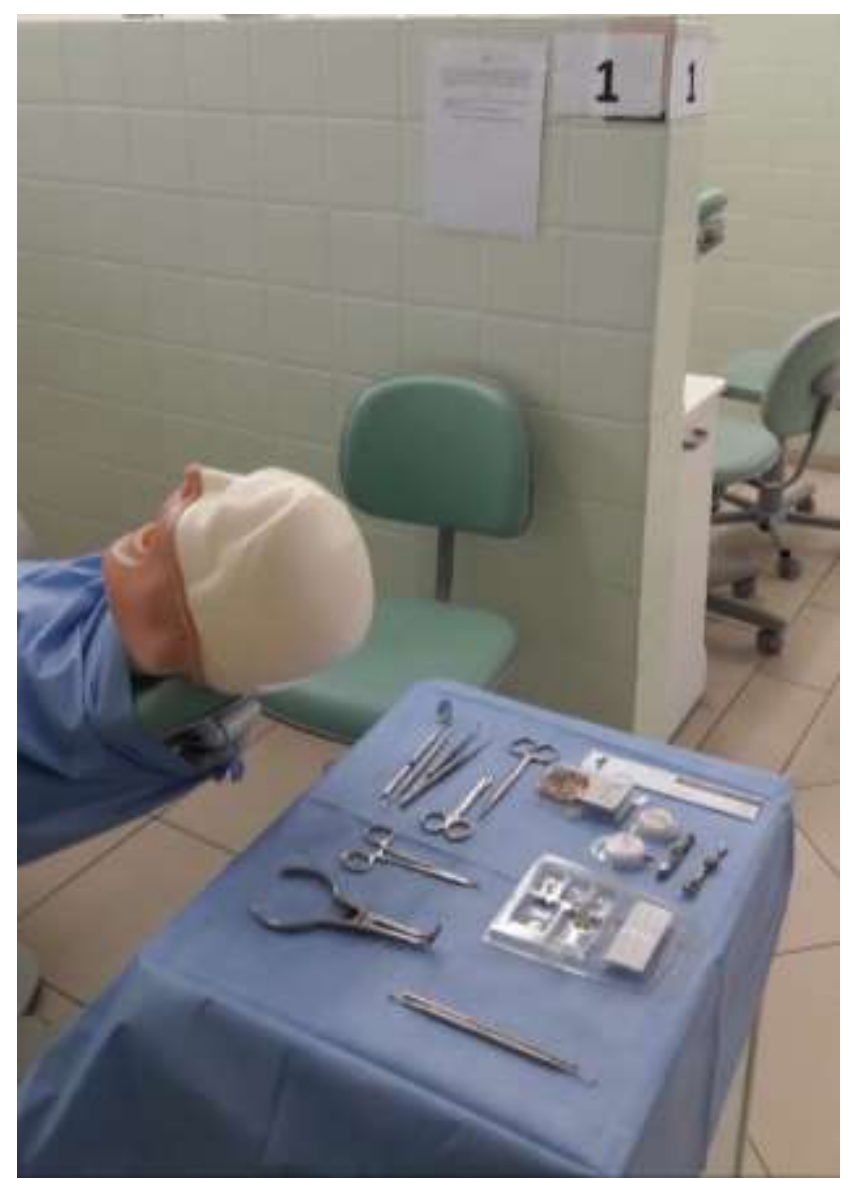

Figura 3. Estação montada para o OSCE

No dia do exame os itens verificados foram: - sinalização do fluxo no circuito: para a sinalização foi selecionado um profissional que orientava a posição dos alunos nas estações;

- marcação e sinalização audível do tempo: um profissional marcava o tempo com o cronômetro e depois sinalizava com um apito;
- bebida, comida e descanso para equipe de examinadores e pacientes: depois de aplicar o exame para a metade da turma de alunos foi realizado intervalo de 15 minutos;

- conferência de materiais e equipamentos: os materiais e equipamentos foram conferidos antes do início do OSCE; e

- distribuição do checklist aos avaliadores e ao final da avaliação recolhimento dos formulários devidamente preenchidos.

\section{Programa de ação após término do OSCE}

Após o término do OSCE os alunos foram reunidos para ofeedback da atividade de avaliação estruturada. Neste momento os professores apontam como deveria ser realizada a tarefa proposta e os principais erros cometidos. O importante é que o aluno conheça de forma mais breve possível como foi seu desempenho para reforçar as respostas certas, superar suas deficiências e corrigir seus erros. É importante para que os professores também identifiquem essas deficiências para a correção antes que o estudante ingresse nas disciplinas clinicas do curso.

Além do feedback aos alunos, é importante que os professores de cada área se reúnam para analisar os erros mais recorrentes durante a avaliação e apontem estratégias para reforçar o ensino durante o semestre, principalmente nas disciplinas pré-clínicas, para que cada vez mais os alunos apresentem um bom desempenho no OSCE.

\section{DISCUSSÃO}

A avaliação do desempenho em ambiente clínico desafia educadores de Odontologia para avaliar não somente conhecimentos e habilidades 
técnicas, mas também o profissionalismo, manejo do tempo, pensamento crítico, relações interpessoais e ética profissional.

O OSCE, como método de avaliação clínico e pré-clínico, apresenta algumas vantagens como uma avaliação mais objetiva, seleção de tarefas e competências a serem avaliadas nos alunos, e possibilidade de avaliar habilidades de comunicação aluno/paciente ${ }^{11}$. Mossey et al.$^{28}$ (2001) relatam em seu estudo a dificuldade de avaliação objetiva na clínica, pois os estudantes atendem pacientes diferentes com queixas e problemas diferentes. Desta forma, o OSCE é um tipo de avaliação mais objetiva, pois a mesma estação e tarefa são aplicadas e avaliadas da mesma forma para todos os alunos, por meio de um checklist. Este checklist tem o objetivo de aumentar a precisão na detecção de falhas e oferecer elementos objetivos para dar o feedback aos alunos, pois a pontuação quantifica $o$ desempenho dos alunos nas habilidades avaliadas $^{19,20,27}$.

No presente estudo as pontuações de $0,0,5 \mathrm{e}$ 1 foram utilizadas em 10 quesitos a serem avaliados na estação, mas nada impede que essa pontuação tenha outros valores ou que sejam avaliados mais quesitos, como no estudo de Höfer et al. ${ }^{19}$ (2013) que avaliou de 8 a 14 quesitos em cada estação, com pontuações de 0,1 e 2 .

As habilidades de comunicação puderam ser observadas na realização das estações da Semiologia, nas quais o aluno interagiu com um paciente simulado para obter informações importantes no diagnóstico da doença pela sintomatologia e na abordagem do paciente para a tomada radiográfica na estação de Radiologia. Essa avaliação individual das habilidades de comunicação só é possível com o OSCE, pela dificuldade de acompanhar os alunos individualmente na clínica, como observado por Graham et al. em $2014^{22}$.

O OSCE pode ser usado para avaliação de várias competências e habilidades de conteúdos associados ou isolados ${ }^{22}$. Pode-se avaliar, por exemplo, os componentes curriculares pré-clínicos de um determinado termo ou ano letivo como utilizado em nosso estudo, ou então como relatado por Höfer et al. ${ }^{19}$ (2013) que aplicaram o OSCE em Cirurgia Bucomaxilofacial, com 10 estações avaliando competências e habilidades distintas dessa área.

No entanto, algumas desvantagens do método são o tempo reduzido das estações, a impossibilidade de simular procedimentos invasivos em pacientes reais, ansiedade dos alunos, dificuldade de mobilização de um grande número de examinadores e pacientes, assim como alto custo ${ }^{10,11,21}$.

O tempo das estações foi de 5 minutos de execução e 1 minuto de leitura da estação, pois na aplicação do OSCE, em nosso estudo, o número de alunos era elevado (70 alunos), o que consumiu mais de 9 horas contando o tempo para ofeedback. Alguns autores como Höfer et al. ${ }^{19}$ (2013) e Landes et al. $^{20}$ (2014), realizaram o OSCE com um número maior de estações e sugeriram a possibilidade de 10 minutos em cada estação, já que o número de alunos em seus trabalhos era reduzido.

Apesar de não ter sido realizado nenhum questionário para verificação de ansiedade, foi observado, por relato espontâneo, o nervosismo e a ansiedade de grande parte dos alunos, o que pode ser explicado pelo ambiente da própria avaliação e por ser a primeira vez que eles estavam entrando em contato com esse tipo de exame. No entanto, 
relataram que o OSCE é uma avaliação mais objetiva e aproximada da realidade de atividade clínica, o que corrobora com a literatura ${ }^{22,28}$.

Uma dificuldade encontrada na aplicação do OSCE foi a mobilização dos avaliadores, no total de 14 , pois foi proposta a presença de uma dupla de avaliadores para cada uma das 7 estações. Höfer et al. ${ }^{19}$ (2013) sugerem que seja utilizado um avaliador por estação para minimizar a quantidade de avaliadores.

A maior desvantagem do OSCE relatada pela maioria dos autores e pela experiência deste estudo é a impossibilidade da realização de procedimentos invasivos em pacientes reais ficando estes para serem avaliados nas disciplinas clínicas com outro método de avaliação ${ }^{11,21,24,26-28}$.

A etapa final da realização do OSCE, e uma das mais importantes, é o feedback, que tem como objetivo observar a performance do aluno na estação, gerando informações para comparação entre o aprendizado demonstrado e o aprendizado esperado. Discutir essas informações para que o aluno possa confirmar, adicionar, substituir, ajustar ou reestruturar o conhecimento na memória é praticar o feedback. Este retorno deve ser associado a uma autoavaliação do aluno. O feedback pode ser realizado ao final da estação com um tempo estipulado antes do rodízio ou ao final do OSCE. Importa que o aluno conheça de forma mais breve possível quais foram seus acertos e erros para reforçar as respostas certas, superar suas deficiências e corrigir seus erros. Além disso, no planejamento do OSCE, é interessante incluir o tempo de feedback no cálculo do tempo total do exame 9,21,23.

\section{CONSIDERAÇÕES FINAIS}

O OSCE pode ser considerado uma técnica de avaliação adequada para o alcance de competências clínicas na formação de cirurgiõesdentistas, por proporcionar ao aluno de graduação a vivência de atividades similares à realidade que enfrentarão no atendimento clínico e em sua futura atuação profissional. Esse método de avaliação propicia aos estudantes oportunidade de serem avaliados de uma forma mais objetiva e poder aprender com o feedback do seu desempenho no exame.

\section{ABSTRACT \\ OSCE in Dental clinical evaluation: the report of an experience with undergraduate students}

The Objective and Structured Clinical Examination (OSCE) is an exam organized in stations that simulate clinical reality and students are evaluated in specific tasks such as anamnesis, physical examination, communication with patients, noninvasive clinical procedures, among others. Students rotated through stations, and one or two examiners score their performance in each task, with predetermined criteria and time. The objective of this study was to report on the experience of applying the OSCE in undergraduate and how to structure it. The first OSCE applied for Dental undergraduate students at Universidade do Oeste Paulista (UNOESTE) was in 2016, with the objective of evaluating the competencies of the students who would enter the clinics in the following semester. The evaluation was applied to 70 students at the end of the third year and the contents selected were Restorative Dentistry, Periodontics, Endodontics, Radiology, Biosafety, Anesthesiology and Oral Diagnosis. The OSCE can be considered an adequate evaluation technique for the attainment of clinical competences in the training of dental surgeons, as it provides the undergraduate student with the experience of activities similar to the reality he will face in clinical care and his future professional performance. This assessment method gives students the opportunity to be evaluated more 
objectively and to learn from feedback on their exam performance.

Descriptors: Assessment. Dental Education.

Dentistry.

\section{REFERÊNCIAS}

1. Brasil. Ministério da Educação. Conselho Nacional de Educação, Câmara de Educação Superior. Diretrizes Curriculares Nacionais do Curso de Graduação em Medicina. Resolução CNE/CES 4/2001.

2. Steiner-Oliveira C, Kantovitz KR, Teixeira MS, Vieira KA, Serra MD, Gambareli FR et al. Estudo preliminar do instrumento de avaliação clínico proposto aos alunos de graduação de odontopediatria da Faculdade de Odontologia de Piracicaba - Unicamp. Ciênc Odontol Bras. 2006; 9(1):77-86.

3. Miller, GE. The assessment of clinical skills/competence/performance. Acad Med. 1990; 65(9 Suppl):S63-7.

4. Amaral FTV, Troncon LEA. Participação de estudantes de medicina como avaliadores em exame estruturado de habilidades clínicas (OSCE) Rev Bras Educ Med. 2007;31(1):81-9.

5. Wass V, Van der Vleuten C; Shatzer,J, Jones, R. Assessment of clinical competence. Lancet. 2001;357(9260):945-9.

6. Manogue M, Brown G. Developing and implementing an OSCE in dentistry. Eur J Dent Educ. 1998;2:51-7.

7. Galato D, Alano GM, França TF, Vieira AC. Exame Clínico Objetivo Estruturado (ECOE): uma experiência de ensino por meio de simulação do atendimento farmacêutico. Interface Comum Saúde Educ. 2011;15(36):309-20.
8. Medeiros SB, Pereira CDFD, Tourinho FSV, Fernandes LGG, Santos VEP. Exame clínico objetivo estruturado: reflexões sob um olhar da enfermagem. Cogitare Enferm. 2014; 19(1):170-73.

9. Eberhard L, Hassel A, Bäumer A, Becker F, Beck-Mubotter J, Bömicke W, et al. Analysis of quality and feasibility of an objective structured clinical examination (OSCE) in preclinical dental education. Eur J Dent Educ. 2011;15(3):172-8.

10. Graham R, Zubiaurre Bitzer LA, Anderson OR. Reliability and predictive validity of a comprehensive preclinical OSCE in Dental Education. J Dent Educ. 2013;77(2):161-7.

11. Albino JE, Young SK, Neumann LM, Kramer GA, Andrieu SC, Henson L, Horn B, Hendricson WD. Assessing dental students' competence: best practice recommendations in the performance assessment literature and investigation of current practices in predoctoral dental education. J Dent Educ. 2008;72(12):1405-35.

12. Kupke J, Wicht MJ, Stützer H, Derman $\mathrm{SH}$, Lichtenstein NV, Noack MJ. Does the use of a visualised decision board by undergraduate students during shared decision-making enhance patients' knowledge and satisfaction? A randomised controlled trial. Eur J Dent Educ. 2013; 17(1):19-25.

13. Näpänkangas R, Karaharju-Suvanto $T$, Pyörälä E, Harila V, Ollila P, Lähdesmäki R, Lahti S. Can the results of the OSCE predict the results of clinical assessment in dental education? Eur J Dent Educ. 2016; 20(1):3-8.

14. Romito L, Schrader S, Zahl D. Using 
Experiential Learning and OSCEs to teach and assess tobacco dependence education with first-year Dental students. J Dent Educ. 2014;78(5):703-13.

15. Lele M. A mini-OSCE for formative assessment of diagnostic and radiographic skills at a Dental College in India. J Dent Educ. 2011;75(12):1583-9.

16. Näpänkangas $R$, Harila V, Lahti S. Experiences in adding multiple-choice questions to an objective structural clinical examination (OSCE) in undergraduate dental education. Eur J Dent Educ. 2012; 16(1) :e146-50.

17. Park SE, Kim A, Kristiansen J, Karimbux NY. The influence of examiner type on Dental students' OSCE scores. J Dent Educ. 2015;79(1):89-94.

18. Macluskey M, Durham J, Balmer C, Bell A, Cowpe J, Dawson L, et al. Dental student suturing skills: a multicentre trial of a checklist-based assessment. Eur J Dent Educ. 2011;15(4):244-9.

19. Höfer SH, Schuebel F, Sader R, Landes C. Development and implementation of an objective structured clinical examination (OSCE) in CMF surgery for dental students. J Craniomaxillofac Surg. 2013; 41(5):412-6.

20. Landes C, Höfer, S H, Shuebel F, Ballon A, Teiler A, Tran A, Weber A, et al. Longterm, prospective teaching effectivity of practical skills training and first OSCE in cranio maxillo facial surgery for dental students. J Craniomaxillofac Surg. 2014; 42(1):e97-110.

21. Barman A. Critiques on the objective structured clinical examination. Ann Acad
Med Singapore. 2005;34(8):478-82.

22. Graham R, Zubiaurre Bitzer LA, Mensah FM, Anderson OR. Dental student perceptions of the educational value of a comprehensive, multidisciplinary OSCE. J Dent Educ. 2014 May;78(5):694-702.

23. Amaral E, Domingues RCL, Zeferino AMB. Avaliando competência clínica: o método de Avaliação Estruturada Observacional. Rev Bras Educ Méd. 2007; 31(3):287-90.

24. Takahashi EAS, Jorge MEN. Ensino e aprendizagem de habilidades afetivas do profissional de saúde. Colloq Human. 2013; 10:911-8.

25. Prislin MD, Fitzpatrick CF, Lie D, Giglio M, Radecki S, Lewis E. Use of an objective structured clinical examination in evaluating clinical performance. Fam Med. 1998;30 (5):338-44.

26. Nunes SOB, Muraguchi EMO, Ferreira Filho OF, Pontes RMA, Cardoso LTQ, Grion CMC, et al. O Ensino de habilidades e atitudes: um relato de experiências. Rev Bras Educ Méd. 2013;37(1):126-31.

27. Harden RM, Stevenson M Downe WW, Wilson GM. assessment of clinical competence using Objective Structured Examination. Brit Med J 1975;22(1):447-51.

28. Mossey PA, Newton JP, Stirrups DR. Scope of the OSCE in the assessment of clinical skills in dentistry. Br Dent J. 2001 24;190(6):323-6.

Correspondência para:

Gustavo de Almeida Logar

e-mail: gustavologar@unoeste.br

Av. José Campos do Amaral 227 casa 43

190.510-80 Presidente Prudente/SP 